\title{
Scout Scan
}

National Cancer Institute

\section{Source}

National Cancer Institute. Scout Scan. NCI Thesaurus. Code C94979.

An initial scan with only the minimal detail needed to select the desired range of bed positions to be used in subsequent scans. 